\title{
On steering wobble oscillations of motorcycles
}

\author{
R S Sharp* and D J N Limebeer \\ Department of Electrical and Electronic Engineering, Imperial College London, UK
}

\begin{abstract}
The paper is aimed at an improved understanding of steering wobble oscillations of motorcycles through simulation. The background to the problem is discussed first. Then, an existing mathematical model of a manoeuvring motorcycle and rider is extended to include a yaw freedom for the upper body of the rider. The rider upper body and arm structural parameters are chosen in the light of newly published results from the testing of human subjects in a driving simulator, with forced motion of the steering wheel by means of an electric motor. Results show that steering wobble oscillations grow more vigorously as their amplitude increases beyond a few degrees of steering and that the stabilizing influence of the rider's tensing his/her muscles in response to a growing wobble problem is small. The work supports the idea that any machine which has a very lightly damped wobble mode at some operating condition may be made unstable by an unusual set of initial conditions and that the natural response of the rider to the problem will be largely ineffective. This idea is closely aligned with anecdotal accounts from general motorcycle usage.
\end{abstract}

Keywords: motorcycle, dynamics, steering wobble, oscillation, stability, human factors

\section{INTRODUCTION}

Early effective motorcycle dynamics models presumed two rigid frames pin-jointed together at the steering head. The rider's mass constituted part of the rear frame [1]. Such models predict the likelihood of weave and wobble oscillations, both at high speeds. The models, being linear, predict relatively benign unstable behaviour, under appropriate design and running conditions. For constant-speed running, the modal damping decreases as the speed increases and oscillations grow rather slowly if the speed is only a little beyond critical. The predicted behaviour is progressive and, to that extent, is not particularly dangerous. In practice, weave oscillations largely follow this predicted pattern, but the wobble mode mostly does not.

Experiments carried out by Eaton [2], Roe and Thorpe [3], Verma [4] and Weir and Zellner [5] showed the wobble mode frequency to be predicted quite accurately, but the damping at moderate speeds of travel to be much less than forecast. The Dunlop Tyre Company made a film in the late 1970s demonstrating low-amplitude sustained wobbling at modest speeds, with the rider's hands off the handlebars, consistent with

The MS was received on 1 March 2004 and was accepted after revision for publication on 13 August 2004.

* Corresponding author: Department of Electrical and Electronic Engineering, Imperial College London, Exhibition Road, London SW7 2AZ,UK.E-mail: robin.sharp@ic.ac.uk the measurements recorded in references [2] to [5]. Inclusion of a structural flexibility, representing twisting of the rear frame at the steering head about an axis perpendicular to the steer axis or lateral bending of the front forks, brought theoretical predictions into line with the above observations $[6,7]$ but also predicted much higher wobble mode damping than before at high speeds. Thus, models containing a lumped frame torsional compliance predict least damping of the wobble mode at moderate speeds for low stiffnesses and at high speeds for high stiffnesses. How best to represent the distributed compliance of a real machine in a lumped-parameter model remains a research issue [8].

Many less scientific accounts, some cited in reference [9], and a plethora of anecdotes [10] testify to the existence of the 'tank slapper'. This is a violent form of the wobble oscillation that may occur at any speed above about $10 \mathrm{~m} / \mathrm{s}$, depending on the machine and its condition (it is also known to occur occasionally with bicycles at what for them is high speed). It generally is triggered by an initial event, or a succession of regular smaller events [road undulations tuned to the wobble frequency (see, for example, reference [9])] and it quickly develops into a limit stop to limit stop vibration, despite the best efforts of the rider to resist the action. Clearly, the tank slapper is dangerous and a fuller account of it is needed.

Although the frequency of the wobble is primarily determined by the trail, the front tyre cornering stiffness and the steering system inertia (www.ee.ic.ac.uk/ 
control/motorcycles), its stability is substantially affected by the support conditions provided by the steering head, i.e. the boundary conditions $[\mathbf{6}, \mathbf{7}]$. Steering damping is known both from practice and from theory to control the problem [9] but this may be unsatisfactory from other viewpoints (in particular, it usually destabilizes the weave mode). Other influences are the frame compliance, as discussed above, and the dynamics of the rider. Rider compliance has been included in advanced models for some time now [11-14], but there has been little wisdom regarding the choice of parameter values for stiffness and damping. Experimental results obtained by Nishimi et al. [15] in laboratory shaker testing of human subjects suggested that the rider should be allowed a lateral translational freedom of the hips and an upper body rotation in roll relative to the hips. They identified appropriate parameter values. This basis was used in reference [13] but it has not become popular. It is employed here, except that the hips' lateral freedom is omitted as being at variance with common observations. The consequence is that the rider's lean motion, decoupled from the motorcycle dynamics, has a natural frequency of $1.27 \mathrm{~Hz}$ and a damping factor of 0.485 .

Recent experimental results on the frequency response properties of human subjects holding steering wheels [16] provide a foundation for modelling the contribution to the steering system of a rider, firstly, in a relaxed state and, secondly, trying to suppress handlebar vibrations. The new results also allow estimation of the rider's upper body restraint properties, so that a more complete modelling of steering wobble oscillations than before becomes possible. In section 2 , an existing motorcycle dynamics model $[\mathbf{1 7}, \mathbf{1 8}]$ is extended to include an additional yaw freedom for the rider's upper body and parameter values to represent relaxed or tense riding are considered. In section 3 , it is shown that the wobble mode of the standard machine and relaxed rider can be destabilized by reducing the effectiveness of the steering damper and simulations are used to indicate that, if the parameters of the relaxed state model are chosen to make the wobble mode somewhat divergent, the rate of growth increases with increasing amplitude. Simulation results also show that muscle pre-tensioning by the rider stabilizes the wobble only a little and the implications of the rider's tensing as a response to a wobble motion developing are studied. In section 4, conclusions relating to the occurrence of 'tank slapper' oscillations are drawn.

\section{MATHEMATICAL MODEL ENHANCEMENT}

A motorcycle multibody dynamics model was described in detail in reference [17], the account of which has been updated and extended in [18]. Sections covered machine geometry, masses and inertias, stiffness and damping properties, tyre-ground contact geometry, tyre forces and moments and the 'monoshock' rear suspension. The upper body of the rider, making a significant contribution to the mass of the rear frame, was allowed a roll freedom relative to that frame but it was pointed out that no conventional wisdom is available for the definition of the stiffness and damping properties of the upper body's support system. Fortunately, the parameters appear to be non-critical. However, as stated above, the stability of the steering wobble mode is strongly influenced by the boundary conditions imposed on the front frame at the steering head, so that the rider's motions during a wobble will feed back into the boundary conditions and contribute to determining the stability. Especially when the rider tenses as a result of sensing the onset of dangerous oscillations, there is a closed structural loop, in which the rider tries to control the steering vibrations with his/her arms, the forces in the arms feed back into the shoulders, the upper body participates in the vibrations and the mobility at the steering head is affected. To model this loop closure realistically, an additional yaw freedom for the rider's upper body is needed (Fig. 1). Parameter values for the various contributions from the rider are also necessary.

Experiments reported in reference [16] involved the measurement of steering wheel rotations and torques in response to filtered pseudo-random binary-sequence excitation of such rotations through the steering column of a driving simulator. The 'driver' was required to adopt each of two strategies, the first involving minimal gripping of the steering wheel while avoiding sliding of hands relative to wheel, and the second to minimize the motions of the wheel by pre-tensioning and thereby stiffening relevant muscles. It was found that excitation amplitude variations made no significant differences to the frequency response properties identified (linear behaviour) and that the driver contributes inertia, damping and stiffness to the steering wheel. The inertia was substantially the same, independent of the muscular strategy used. With pre-tensioning, some damping was added and the stiffness was raised markedly compared with the passive case.

The relationship between motorcycle rider and handlebars is seen as very similar to that between driver and steering wheel in the simulator, so that a direct application of the parameter values identified is reasonable. Further, the results indicate that the pre-tensioned arm muscles provide only moderate stiffness in absolute terms and very little damping. The results can be extrapolated to yield the rider's upper body restraint stiffness and damping values substantially lower than have often been presumed, aligning well with results from Nishimi et al. [15].

Thus, in the revised motorcycle-rider model, the rider's upper body has both yaw and roll freedoms relative to the main frame. The rider's arms contribute 


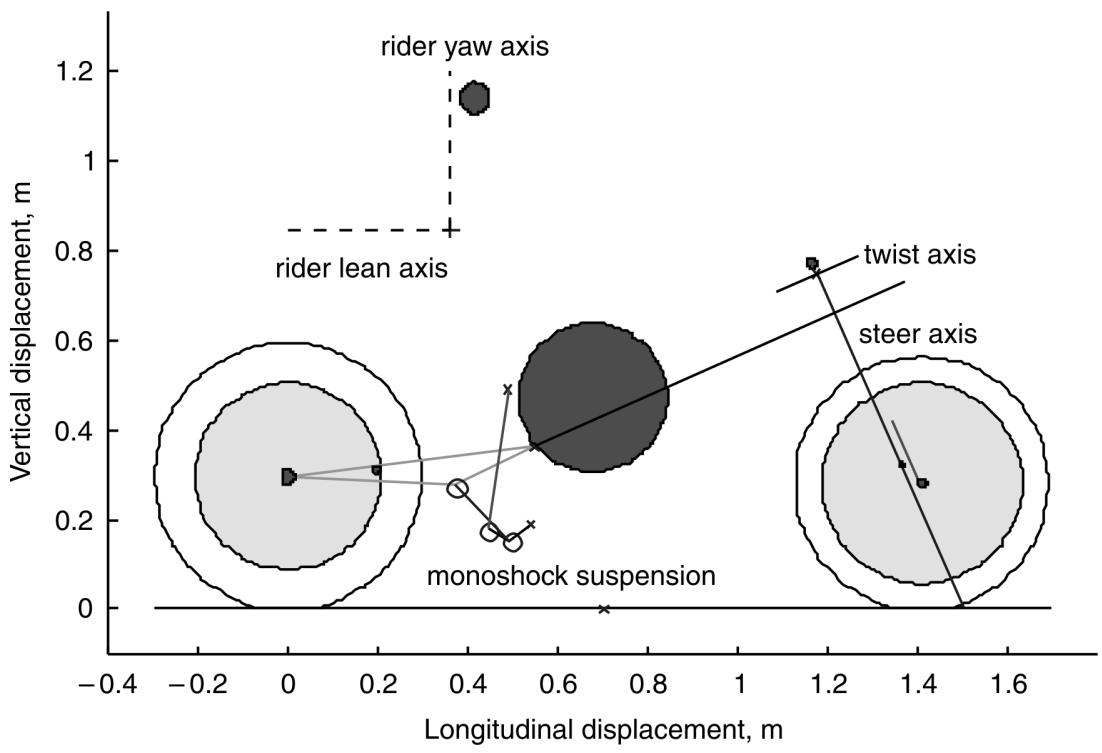

Fig. 1 Diagrammatic motorcycle model, showing the rider's upper body freedoms, in particular. Masses are shown by circles, with the radius proportional to the mass itself

modestly to the steering inertia of the front frame and a parallel rotational stiffness and damping element acts between the handlebars and the rider's upper body. Following reference [16], the steering inertia contribution is fixed at $0.103 \mathrm{~kg} \mathrm{~m}^{2}$, while the steering stiffness and damping parameters are $3.2 \mathrm{Nm} / \mathrm{rad}$ and $0.72 \mathrm{Nms} / \mathrm{rad}$ respectively for relaxed riding and
$60 \mathrm{Nm} / \mathrm{rad}$ and $1.8 \mathrm{Nms} / \mathrm{rad}$ respectively for tense riding. From these values, the rider's upper body restraint parameters are estimated as $60 \mathrm{Nm} / \mathrm{rad}$ and $13.5 \mathrm{~N} \mathrm{~m} \mathrm{~s} / \mathrm{rad}$ in yaw and $380 \mathrm{~N} \mathrm{~m} / \mathrm{rad}$ and $34 \mathrm{~N} \mathrm{~m} \mathrm{~s} / \mathrm{rad}$ in roll for relaxed riding and as $120 \mathrm{Nm} / \mathrm{rad}$ and 13.5 $\mathrm{Nms} / \mathrm{rad}$ in yaw and $760 \mathrm{Nm} / \mathrm{rad}$ and $34 \mathrm{Nms} / \mathrm{rad}$ in roll for tense riding.

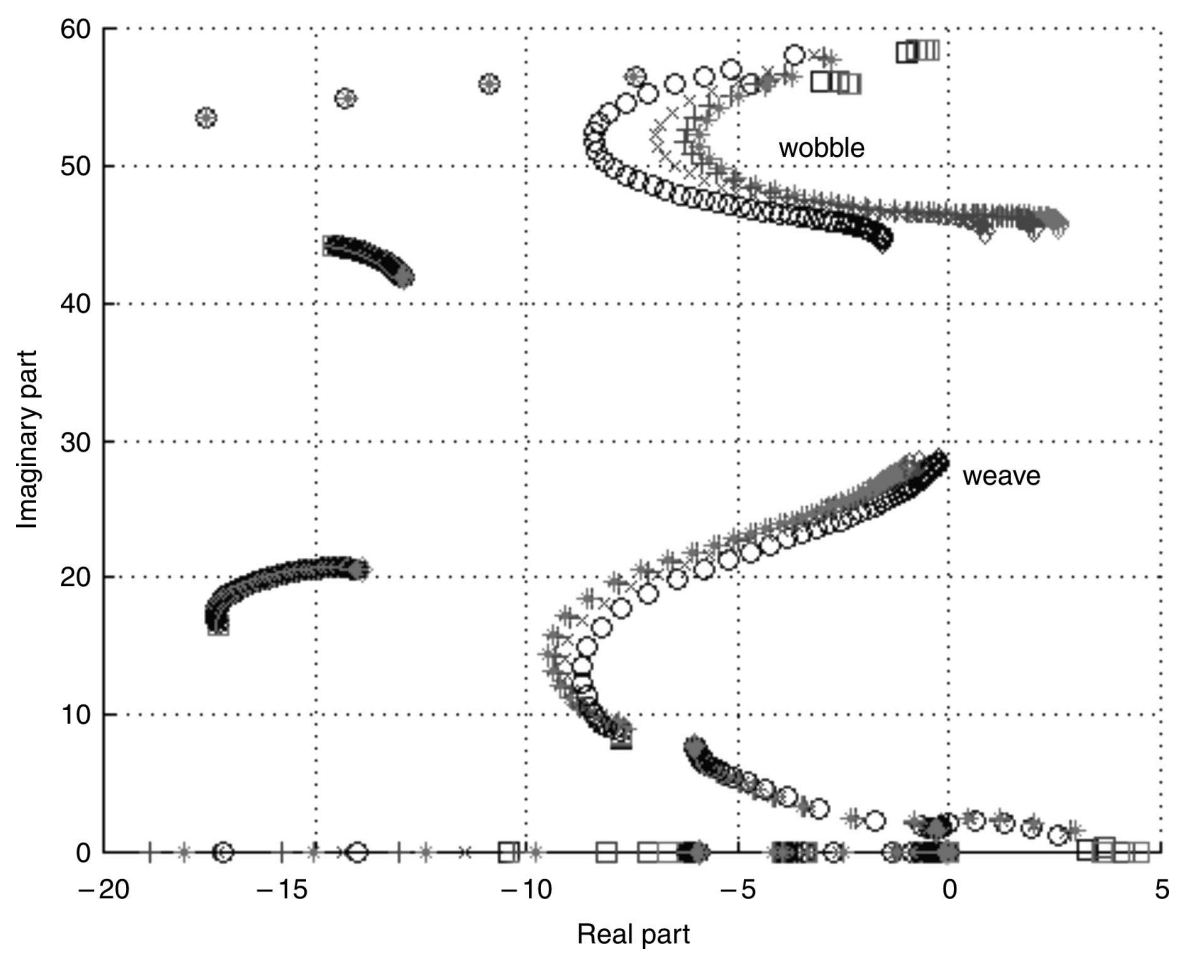

Fig. 2 Root loci for motorcycle and relaxed rider in straight running through a speed range from $0.1 \mathrm{~m} / \mathrm{s}(\square)$ to $70.1 \mathrm{~m} / \mathrm{s}(\diamond)$ as the steer damping changes from 6.944 to $0.868(\mathrm{O}, \times,+, *)$ in a geometric sequence 


\section{MODAL AND SIMULATION RESULTS}

The stability of the wobble mode of the standard machine at high speed depends on the steering damper, fitted as original equipment (Fig. 2). In order to see how the oscillations grow from a small initial condition, in an unstable case, the steer damping is reduced to $1.736 \mathrm{Nm} \mathrm{s} / \mathrm{rad}$ (one-quarter of the standard value) and the motorcycle is made to run straight at a speed of $50 \mathrm{~m} / \mathrm{s}$, with a very small initial steer angle. The steer angle-time history is shown in Fig. 3 and the ratio of successive swing amplitudes, including both positive and negative swings, is shown in Fig. 4. Mathematically linear behaviour would yield a constant amplitude ratio but the rate of growth of the oscillations increases significantly when the amplitude reaches about $4^{\circ}$. The saturation of the tyre force system is quite sharply destabilizing in respect of the steering wobble mode.
The steering oscillations are accompanied by a number of other motions, according to the eigenvector for the mode (Fig. 5). The upper body motions of the rider, denoted ' $u b$ yaw' and 'ub roll', are significant components of the mode. The lateral motion shown is that of the centre of the main body mass. The open diamond labelled twist is the frame twist angle at the steering head. Since all the calculations are made in SI units, $1 \mathrm{~m}$ corresponds to $1 \mathrm{rad}$ in the diagram.

On repeating the trial in Fig. 3 using the parameters for the tense rider, the wobble mode is stabilized by the change in the rider's strategy only to the extent that the rate of divergence is roughly halved (Fig. 6). A similar increase in the growth rate for larger steer angles is in evidence (Fig. 7). The eigenvector for this case is much the same as before (Fig. 8), implying similar high participation for the rider's upper body movements.

It is now imagined that the rider changes from relaxed

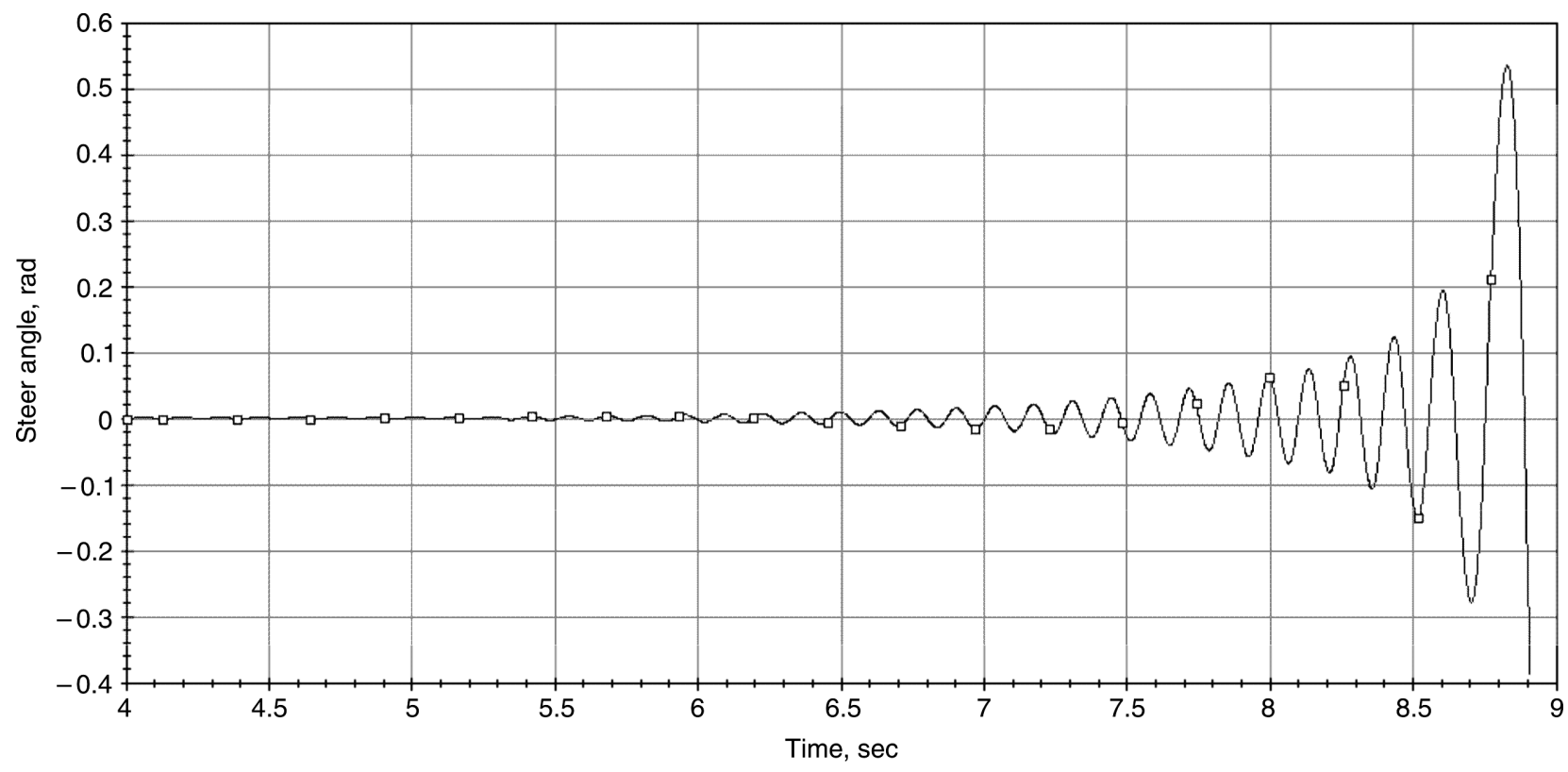

Fig. 3 Later stages of steer angle growth from small initial condition at $50 \mathrm{~m} / \mathrm{s}$ with steer damping coefficient reduced to $1.736 \mathrm{~N} \mathrm{~m} \mathrm{s/rad}$ and relaxed rider parameters

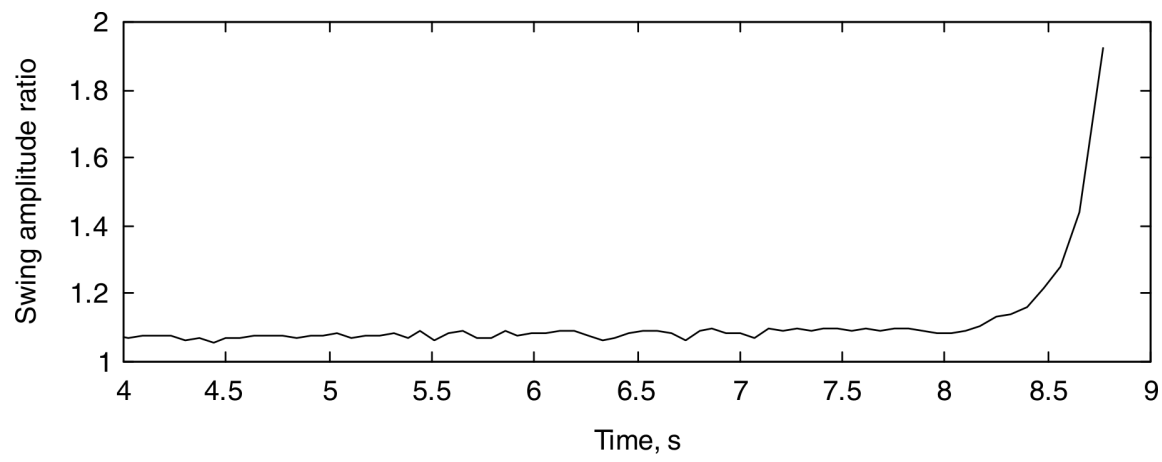

Fig. 4 Ratio of amplitudes of successive positive and negative steer angle peaks for the simulation run in Fig. 3 


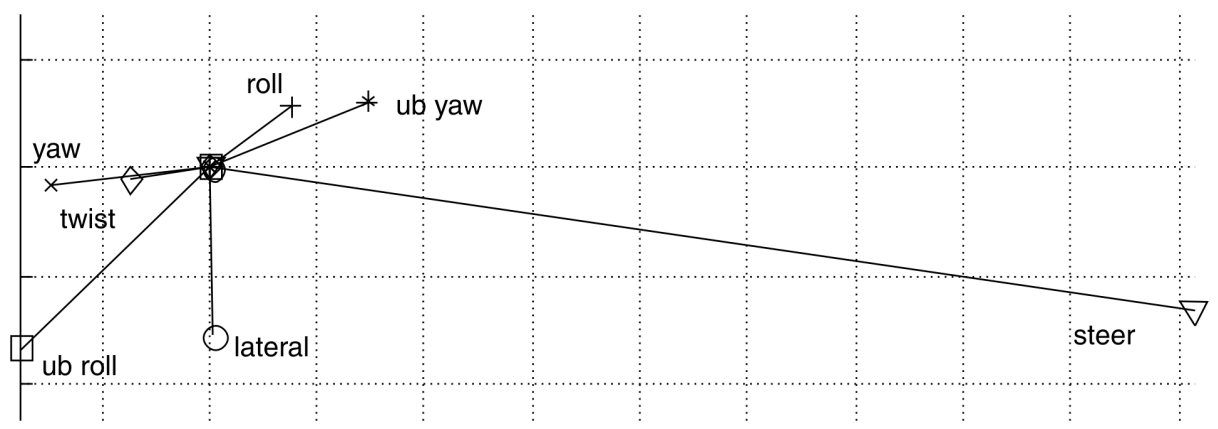

Fig. 5 Wobble mode eigenvector for straight running at $50 \mathrm{~m} / \mathrm{s}$ with parameters for a relaxed rider

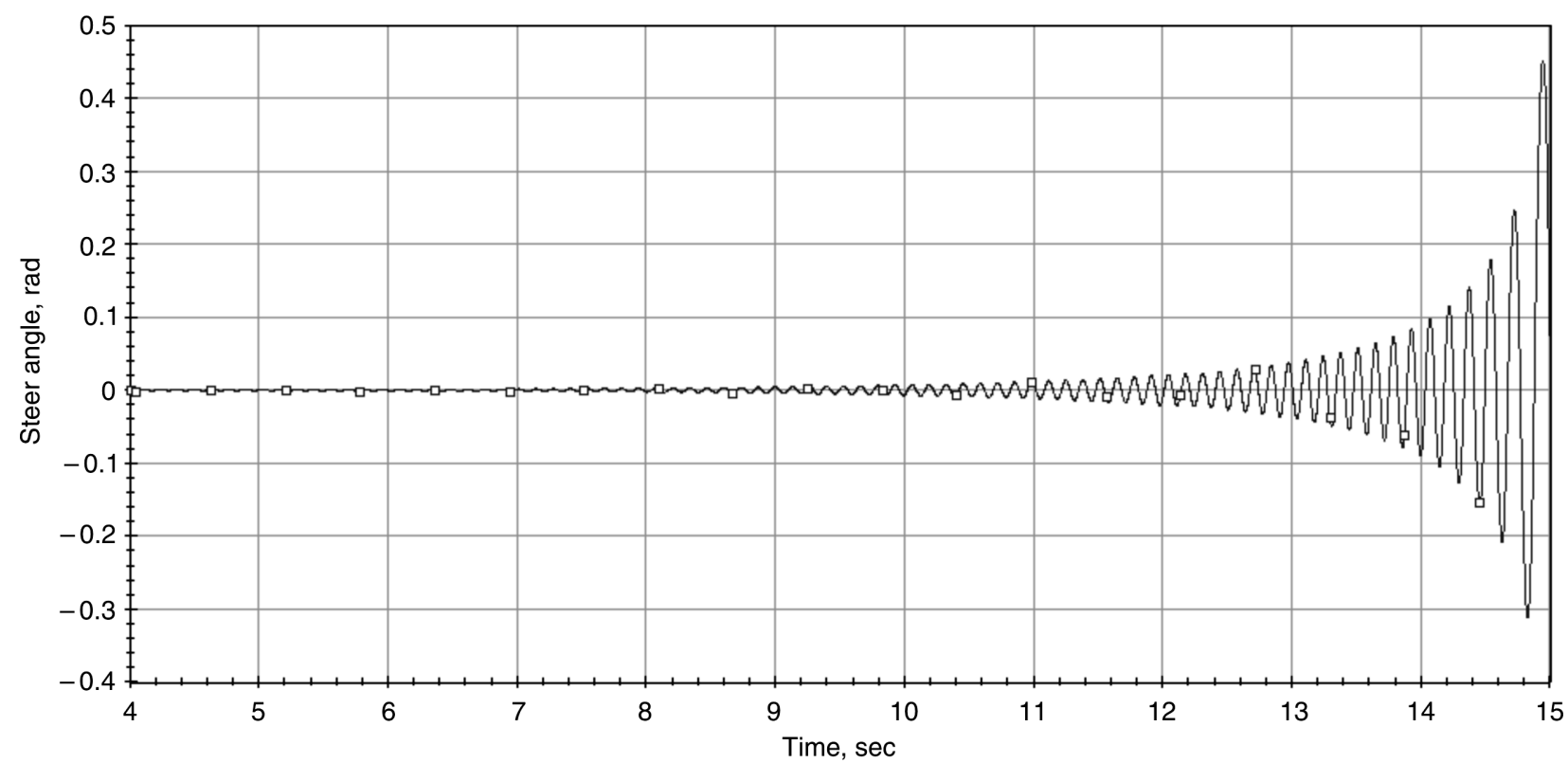

Fig. 6 Later stages of steer angle growth from a small initial condition at $50 \mathrm{~m} / \mathrm{s}$ with the steer damping coefficient reduced to $1.736 \mathrm{~N} \mathrm{~m} \mathrm{~s} / \mathrm{rad}$ and parameters for a tense rider

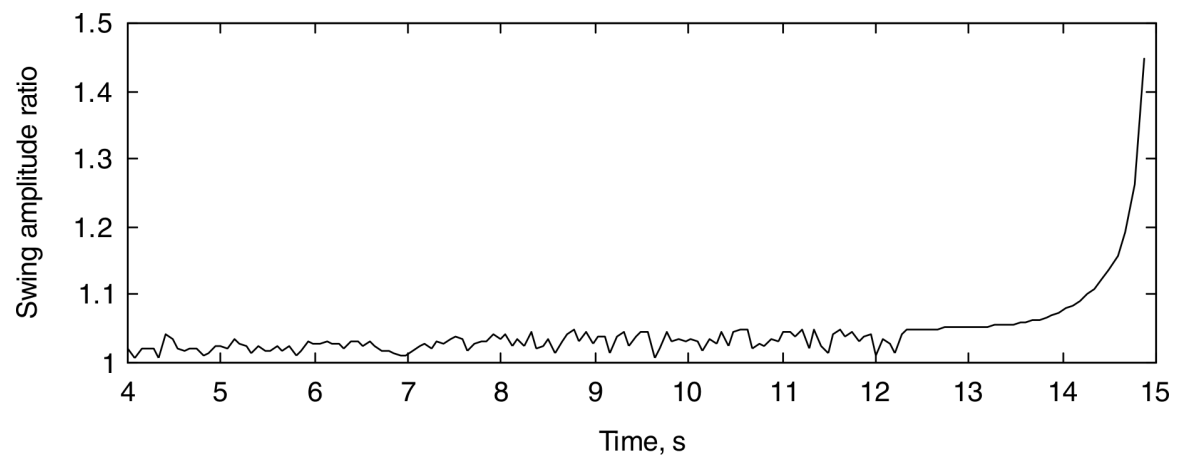

Fig. 7 Ratio of amplitudes of successive positive and negative steer angle peaks for the simulation run in Fig. 6

to tense in mid-event, worried by the growing oscillations into a change in attitude. The circumstances are simulated by using the state of the motorcycle in Fig. 3 at $7.96 \mathrm{~s}$ as the initial condition for a run in which the rider is tense, the results being shown in Figs 9 and 10. The timing of the sudden switching of the rider's parameters implies that it occurs when the stored energy in the 'steering spring' is zero, so that no addition of energy to the system by the spring stiffening is implied. This is not exactly the case in respect of the rider's yaw and roll displacements at the point of switching, implying that some energy is added to the system 


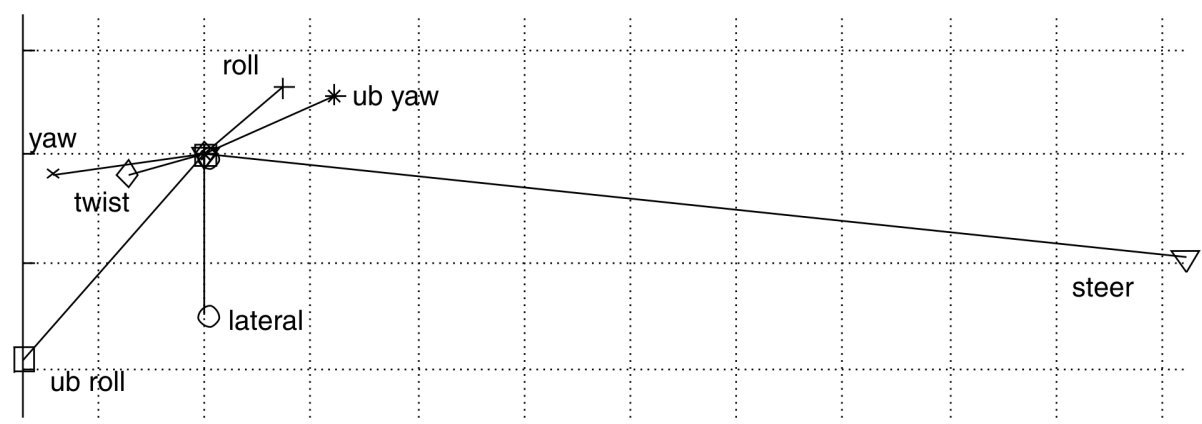

Fig. 8 Wobble mode eigenvector for straight running at $50 \mathrm{~m} / \mathrm{s}$ with parameters for a tense rider

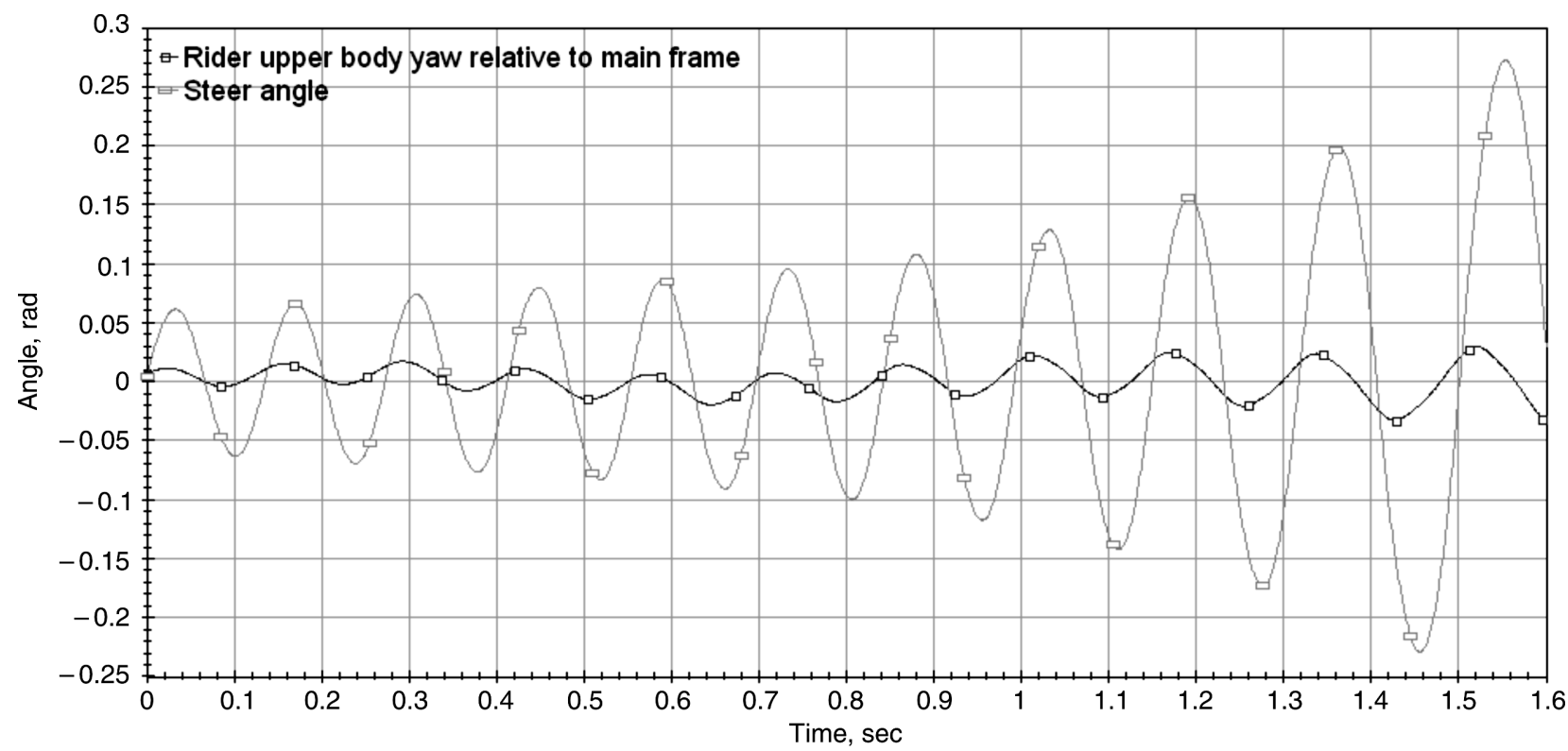

Fig. 9 Rider's upper body relative yaw and steer angle growth from the initial condition corresponding to $7.96 \mathrm{~s}$ into the run in Fig. 3 at $50 \mathrm{~m} / \mathrm{s}$, with parameters for a tense rider

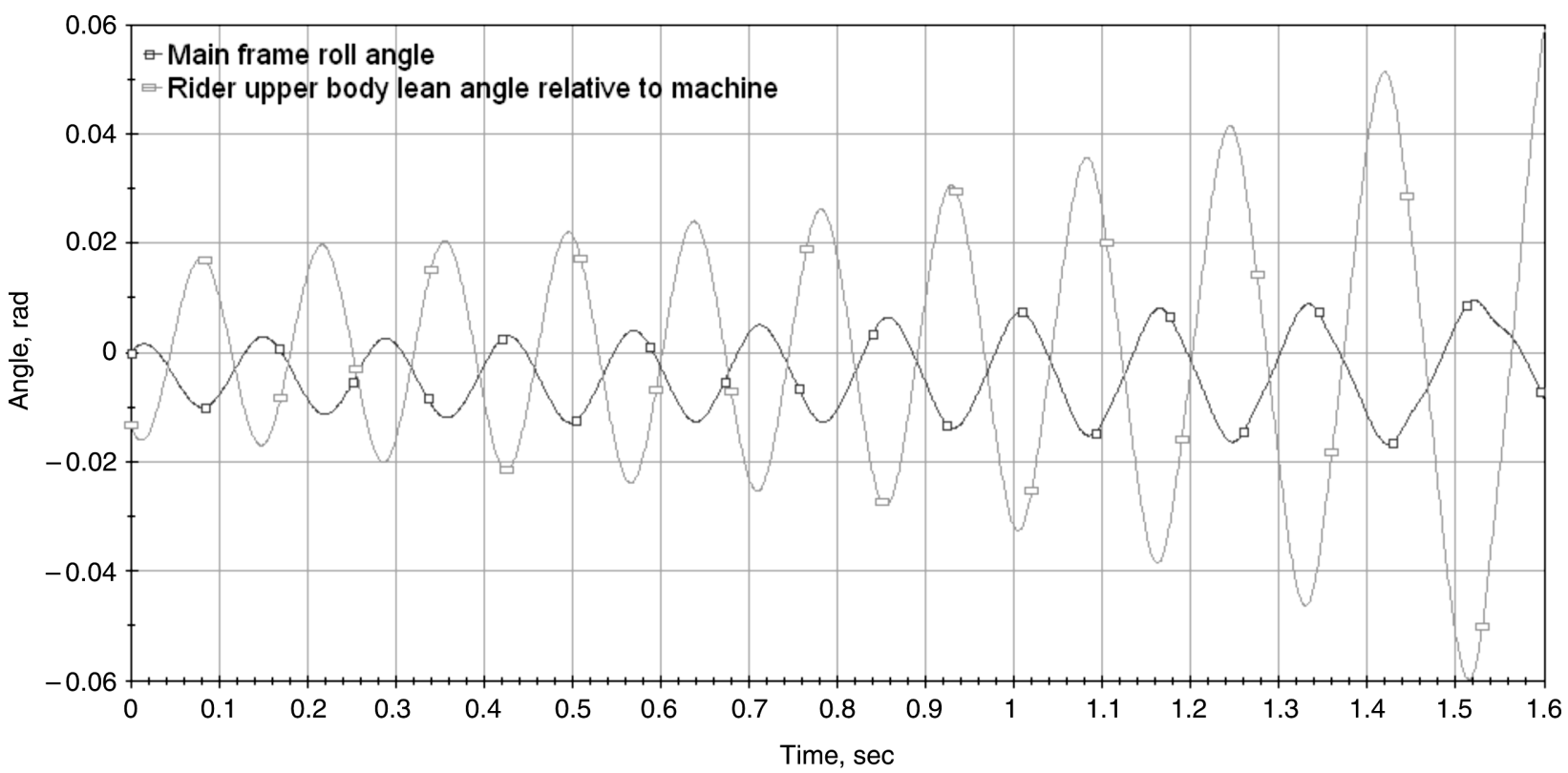

Fig. 10 Motorcycle roll and rider's upper body relative lean angles for the simulation run in Fig. 9 


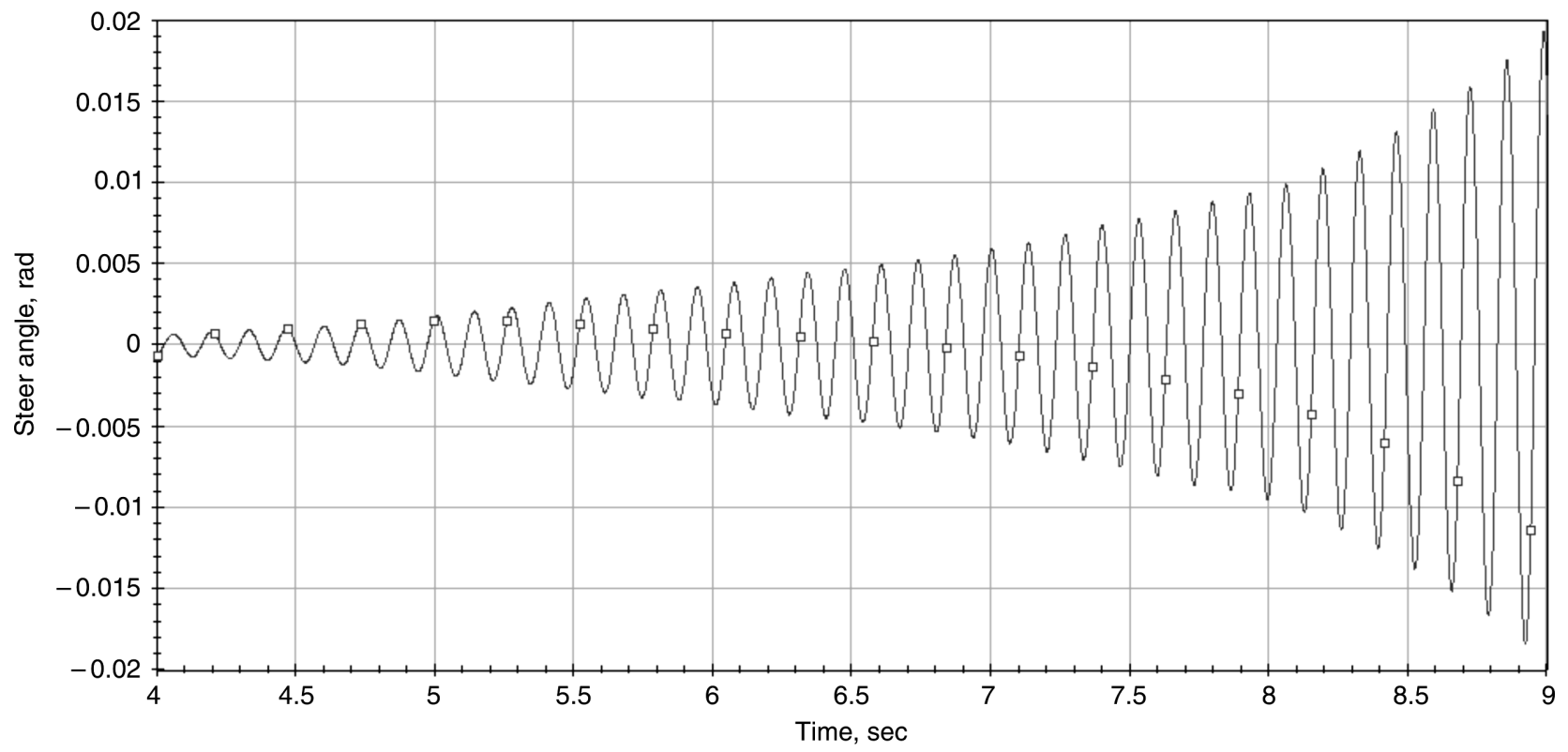

Fig. 11 Segment of steer angle history from simulation run with same initial conditions as for Fig. 3, in which the rider 'stiffens' gradually between 5 and $6 \mathrm{~s}$

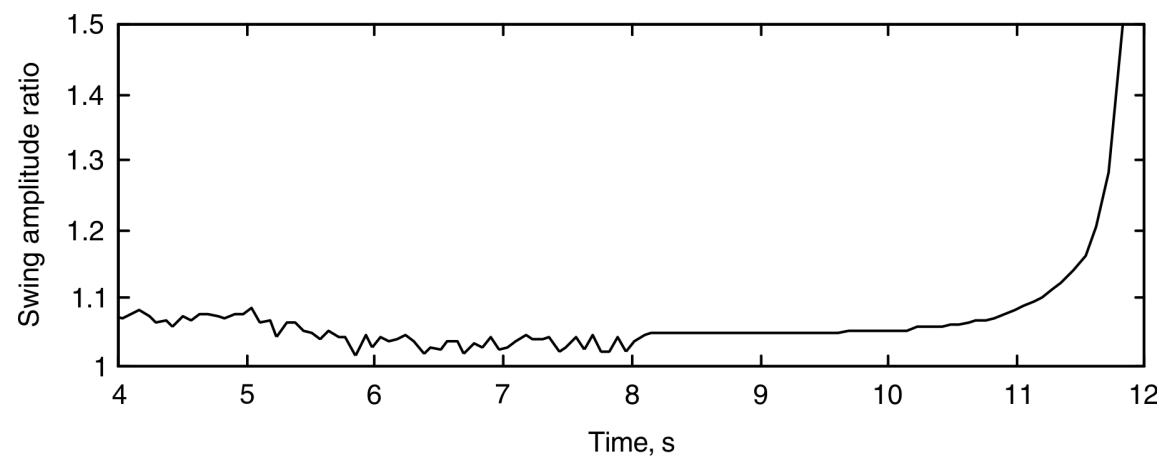

Fig. 12 Ratio of amplitudes of successive positive and negative steer angle peaks for the simulation run in Fig. 11

spuriously by the simplified representation of the rider's actions. The effect is small, however (the added energy is only $35 \mathrm{~mJ}$ ), and the modest stabilizing influence of the rider's tensing his/her muscles is insufficient, in this case, to prevent the oscillations from continuing to grow at a rapid rate, soon to become a limit stop to limit stop vibration. The rider's participation in the motion is again considerable.

An alternative approach to changing the rider's strategy is to do this rather slowly and continuously. The consequence of such an approach is shown in Fig. 11, where the rider changes from relaxed to tense linearly between 5 and $6 \mathrm{~s}$. Again, there is an artificial addition of energy to the system from the stiffening, but it is a small influence. The amplitude growth is shown in Fig. 12, indicating that the extent to which the rider tenses curtailed the rate of growth of the oscillations in the interval 5-6s but failed to prevent further growth. Again, the wobble grows to the steering limit stops, reinforcing the picture developed from Figs 9 and 10.

\section{CONCLUSIONS}

The modelling of the motorcycle rider as a structural component of the man-motorcycle system has been elaborated in such a way that the rider's upper body responds to steering torque fluctuations and thereby affects the steering system boundary conditions. Necessary parameter values have been derived from data from the literature and recent results on the neuromuscular dynamics of human subjects. From the results obtained, the following picture emerges.

The modern stiff-framed motorcycle is prone to highspeed wobble and such a machine may well need a steering damper to stabilize this mode. The mode, when unstable, becomes more rapidly divergent as the steer angle amplitude rises beyond about $4^{\circ}$. Thus, initial conditions involving unusually large departures from equilibrium are likely to lead to growth of wobble oscillations that normally decay, under some operating conditions. Amplitude influences are significant, such 
that, the later any action to stabilize the system is taken, the lower are the chances of success.

The response of the rider to a potential accident situation will primarily be to stiffen both arms and torso, which actions only marginally stabilize the wobble mode. The fundamental difficulty for the rider is that the available stabilizing action is likely to be insufficient to counter a developing problem, especially one started by some unfriendly excitation, in which a relatively large amplitude develops quickly.

The question of how a typical rider can best avoid having a serious wobble problem naturally arises. It is suggested by the results obtained, both here and in prior work, that a motorcycle with a capacity for 'tank slapping' will be a 'head shaker' (to use a vernacular description) over a common range of use. The rider will be in position to identify such behaviour and to employ the knowledge gained to control the usage or to improve the wobble behaviour by increasing the steering damping. However, it is well known $[\mathbf{9}, \mathbf{1 2}, \mathbf{1 3}]$ that increasing steering damping is not a panacea. It may cause other aspects of the steering behaviour to deteriorate, in particular, by destabilizing the high-speed weave motion (see Fig. 2) and/or impeding the motorcycle's selfsteering action at low and moderate speeds.

\section{REFERENCES}

1 Sharp, R. S. The stability and control of motorcycles. J. Mech. Engng Sci., 1971, 13(5), 316-329.

2 Eaton, D. J. Man-machine dynamics in the stabilisation of single track vehicles. Doctoral dissertation, University of Michigan, Ann Arbor, Michigan, 1973.

3 Roe, G. E. and Thorpe, T. E. A solution of the low speed wheel flutter instability in motorcycles. J. Mech. Engng Sci., 1976, 18(2), 57-65.

4 Verma, M. K. Theoretical and experimental investigation of motorcycle dynamics. Doctoral dissertation, University of Michigan, Ann Arbor, Michigan, 1978.

5 Weir, D. H. and Zellner, J. W. Experimental investigation of the transient behaviour of motorcycles. SAE paper 790266, 1979.
6 Sharp, R. S. and Alstead, C. J. The influence of structural flexibilities on the straight running stability of motorcycles. Veh. System Dynamics, 1980, 9(6), 327-357.

7 Spierings, P. T. J. The effects of lateral front fork flexibility on the vibrational modes of straight-running single-track vehicles. Veh. System Dynamics, 1981, 10(1), 21-35.

8 Giles, C. G. and Sharp, R. S. Static and dynamic stiffness and deflection mode measurements on a motorcycle, with particular reference to steering behaviour. In Proceedings of IMechE Conference on Road Vehicle Handling, 1983, pp. 185-192 (Mechanical Engineering Publications Limited, London).

9 Limebeer, D. J. N., Sharp, R. S. and Evangelou, S. Motorcycle steering oscillations due to road profiling. Trans. ASME, J. Appl. Mechanics, November 2002, 69(6), 724-739.

10 Foale, T. Motorcycle handling and chassis design - the art and science; www.tonyfoale.com, 2002.

11 Koenen, C. The dynamic behaviour of a motorcycle when running straight ahead and when cornering. Doctoral dissertation, Technische Universiteit te Delft, Delft, The Netherlands, 1983.

12 Sharp, R. S. Vibrational modes of motorcycles and their design parameter sensitivities. In Vehicle NVH and Refinement, 1994, pp. 107-121 (Mechanical Engineering Publications Limited, London).

13 Sharp, R. S. Stability, control and steering responses of motorcycles. Veh. System Dynamics, May 2001, 35(4-5), 291-318.

14 Pacejka, H. B. Tyre and Vehicle Dynamics, 2002 (Butterworth-Heinemann, Oxford).

15 Nishimi, T., Aoki, A. and Katayama, T. Analysis of straight running stability of motorcycles. In Proceedings of the 10th International Technical Conference on Experimental Safety Vehicles, July 1985, 33 pp.

16 Pick, A. and Cole, D. J. Neuromuscular dynamics and the vehicle steering task. In The Dynamics of Vehicles on Roads and on Tracks (Ed. M. Abe), Supplement to Vehicle System Dynamics, Vol. 41, 2004, pp. 182-191 (Taylor and Francis, London).

17 Sharp, R. S., Evangelou, S. and Limebeer, D. J. N. Improved modelling of motorcycle dynamics. In ECCOMAS Thematic Conference on Multibody Dynamics, Lisbon, Portugal, 1-4 July 2003, MB2003-029, CD-ROM.

18 Sharp, R. S., Evangelou, S. and Limebeer, D. J. N. Advances in the modelling of motorcycle dynamics. Multibody System Dynamics, 2004 (in press). 